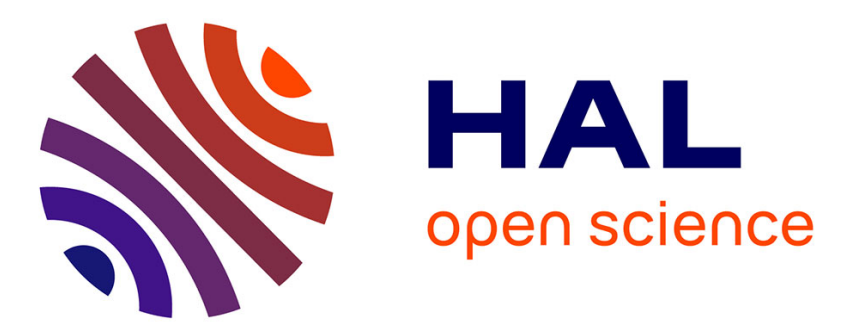

\title{
Synthesis of ab initio and effective Hamiltonian line lists for ozone
}

David Jacquemart, Vladimir Yu. Makhnev, Nikolai F Zobov, Jonathan Tennyson, Oleg L Polyansky

\section{- To cite this version:}

David Jacquemart, Vladimir Yu. Makhnev, Nikolai F Zobov, Jonathan Tennyson, Oleg L Polyansky. Synthesis of ab initio and effective Hamiltonian line lists for ozone. Journal of Quantitative Spectroscopy and Radiative Transfer, 2021, 269, pp.107651. 10.1016/j.jqsrt.2021.107651 . hal-03258734

\section{HAL Id: hal-03258734 https://hal.sorbonne-universite.fr/hal-03258734}

Submitted on 11 Jun 2021

HAL is a multi-disciplinary open access archive for the deposit and dissemination of scientific research documents, whether they are published or not. The documents may come from teaching and research institutions in France or abroad, or from public or private research centers.
L'archive ouverte pluridisciplinaire HAL, est destinée au dépôt et à la diffusion de documents scientifiques de niveau recherche, publiés ou non, émanant des établissements d'enseignement et de recherche français ou étrangers, des laboratoires publics ou privés. 


\title{
Synthesis of $a b$ initio and effective Hamiltonian line lists for ozone
}

\author{
David Jacquemart ${ }^{1}$ \\ Sorbonne Université, CNRS, De la MOlécule aux NAno-objets : Réactivité, Interactions \\ et Spectroscopies, MONARIS, 75005 Paris, France \\ Vladimir Yu. Makhnev, Nikolai F. Zobov \\ Institute of Applied Physics, Russian Academy of Science, Ulyanov Street 46, Nizhny \\ Novgorod, Russia 603950 \\ Jonathan Tennyson \\ Department of Physics and Astronomy, University College London, Gower Street, \\ London WC1E 6BT, United Kingdom \\ Oleg L. Polyansky ${ }^{2}$ \\ Department of Physics and Astronomy, University College London, Gower Street, \\ London WC1E 6BT, United Kingdom; Institute of Applied Physics, Russian Academy of \\ Science, Ulyanov Street 46, Nizhny Novgorod, Russia 603950
}

\begin{abstract}
A synthetic line list for the ozone molecule is presented. Variational calculation using the semi-empirical potential energy surface (PES) and ab initio dipole moment surface (DMS) produce very accurate values of line intensities, but give line positions far away from their experimental values. Furthermore assignment of approximate rotational and vibrational quantum numbers are missing from variationally calculated line list. Effective Hamiltonian calculations are complimentary to ab initio line lists in these properties giving excellent value of line positions, close to experimental ones, and a full set of
\end{abstract}


quantum numbers assignment. The synthesis of both these qualities in one line list is highly desirable. Here a synthetic line list for ${ }^{16} \mathrm{O}_{3}$ is presented. The method of corrections of distorted intensities in variational linelist with $a b$ initio DMS due to the artificial intensity stealing is developed and applied. Comparison of the synthetic line list with all major published line lists and available experimental data is given. The calculated line intensities agree to within experimental error for most bands for which accurate measurements are available.

Keywords:

ozone, potential energy surface, dipole moment surfaces, IR spectra, line intensities, HITRAN database

\section{Introduction}

Ozone, $\mathrm{O}_{3}$, is a much discussed component of our atmosphere. While at low altitudes it is considered an unwanted pollutant which is detrimental to human health, at high altitudes the so-called ozone layer provides essential protection against the harmful effects of solar UV radiation. Atmospheric ozone is therefore regularly monitored [1] with, for example, a number of ground-based and space-borne experiments dedicated to monitoring atmospheric ozone concentrations using spectroscopy $[2,3]$. As a result a number of databases of ozone spectroscopic parameters have been compiled for infrared (IR) $[4,5,6,7,8,9,10]$ and ultraviolet (UV) studies $[9,11]$ which feed into general atmospheric databases such as HITRAN [12] and GEISA [13]. However, monitoring requires high accuracy, in particular intensities for all key bands are needed to better than 1\%, so small relative changes can 
be determined with low uncertainty. In this work we concentrate on the IR spectrum of ozone for which there are well-documented difficulties in determining transition intensities with the accuracy and consistency required for atmospheric studies $[14,9,15]$. This issue has led to a series of recent studies both experimental $[16,17,18]$ and, in a new development for the field, $a b$ initio $[19,20,21]$.

Recent experimental studies [22, 23] have been performed in LERMA using a special $\mathrm{H}$ shaped cell designed to record most intense transitions of ozone at $5 \mu \mathrm{m}$ and $10 \mu \mathrm{m}$ regions. Spectra at 5 and $10 \mu \mathrm{m}$ were recorded simultaneously from the same ozone samples allowing consistent measurements in both spectral regions. Intensities accurate of $1 \%$ or better were measured for around 800 lines. Because of the very good agreement obtained between LERMA measurements and the variational calculation reported in this work, we decided to construct a line list based on assignments and line positions from effective Hamiltonian (EH) calculations but using variational intensities.

In this paper we concentrate on the IR spectrum of ${ }^{16} \mathrm{O}_{3}$. Section 2 describes the calculation of the first principles ozone line list using our previously published semi-empirical potential energy surface (PES) [20] and ab initio the dipole moment surface (DMS), produced by Tyuterev and co-authors [21]. Program DVR3D [24] is used to solve nuclear-motion Schrödinger equation to produce ro-vibrational wavefunctions and to compute line positions and line intensities. Details of the line list calculations are given in this section. Section 3 describes the synthesis of the $a b$ initio line list and the HITRAN2016 [12] line positions obtained from EH calculations. The details 
of line-by-line matching of these two line lists is described. The resulting synthetic line list consists of the EH line positions and the first principles variationally calculated intensities. Section 4 describes the corrections to the ab initio intensities, obtained by comparison with the EH line lists. Since the $\mathrm{EH}$ line positions are more accurate, the information on EH intensities helps to correct outliers, which appear due to intensity stealing between lines affected by resonances [25]. Section 5 presents the results of comparisons of the synthetic line list with all available theoretical calculations and experimental measurements; it demonstrates the significant advantages of the present line list over other available ones. We conclude this paper in the section 6 .

\section{Calculation of the $a b$ initio line list}

The theoretical rotation-vibration line list of any molecule consists of the line position and line intensity, which are calculated as a result of solution of ro-vibrational Schrödinger equation. In order to calculate line positions and line intensities accurately, high precision knowledge of the PES of the molecule and its DMS is necessary. When these two surfaces are available, their use for the calculation of line lists is described elsewhere [26, 27]. Here we will briefly discuss the two surfaces used for our ozone ab initio line list calculation. The ozone PES we have obtained in the [20] as follows. First an ab initio PES was calculated using the MOLPRO package [28] at the multi-reference configuration interaction (MRCI) level of theory and aug-ccpwcVQZ basis set [20]. The accuracy of rovibrational energy levels calculation using this ab initio PES was a few $\mathrm{cm}^{-1}$. A much higher accuracy PES is required to obtain high precision wavefunctions for the calculation of 
intensities [29]. In order to improve the accuracy of the PES the procedure of fitting the starting $a b$ initio PES to the experimental energy levels was used. A very accurate semi-empirical PES was obtained with a standard deviation of the fitted energy levels of only $0.027 \mathrm{~cm}^{-1}[20]$.

The PES describes vibrational levels up to $6000 \mathrm{~cm}^{-1}$. The convergence of energy levels variational calculations was tested up to this limit during its PES [20]. Since the convergence obtained was significantly better than the above mentioned standard deviation of $0.027 \mathrm{~cm}^{-1}$, it was not described in [20]. Tests were performed on the size of the discrete variable representation (DVR) grid, effectively the basis set size, and size of the final Hamiltonian which demonstrated convergence of about $0.002 \mathrm{~cm}^{-1}$ below $6000 \mathrm{~cm}^{-1}$ with the parameters described below. The rotational energies are converged to better than $0.001 \mathrm{~cm}^{-1}$ for $J=5$.

Intensity calculations for lines belonging to the several ozone bands, measured experimentally were also presented in [20]. These calculations used two different DMSs. One ab initio DMS, calculated in [20] and another calculated $a b$ initio by Tyuterev and coworkers [21] at a higher level of theory. The intensities computed these two DMSs differ by a few percent. At the time of the publication of the paper [20] it was unclear which DMS gave the more accurate results. The recent LERMA intensity measurements [22, 23] are very interesting since sub-percent accuracy is obtained in both 5 and $10 \mu \mathrm{m}$ spectral regions thus providing a stringent test of theoretical predictions. As shown below, excellent agreement is obtained with the variational intensities using the DMS of Ref. [21]. For the $\nu_{3}$ and $\nu_{1}+\nu_{3}$ bands at 10 $\mu \mathrm{m}$ and $5 \mu \mathrm{m}$ respectively, the LERMA measured intensities and variational 
intensities agree with a standard deviation (SD) of around $0.5 \%$ (see section 5). The LERMA intensity measurement of around 800 of ${ }^{16} \mathrm{O}_{3}$ lines allows a clear choice to be made in favor of the DMS of paper [21]. In the present variational line list calculation this DMS has been used.

For the line positions and intensities calculation we used the program suite DVR3D [24], making use of an exact kinetic energy operator. Radau coordinates and Morse-like oscillators with parameters $r_{e}=2.8 \mathrm{a}_{0}, D_{e}=0.1 \mathrm{E}_{h}$, $\omega_{e}=0.0024$ a.u. were used in the calculations. 20 DVR radial grid points and 70 angular grid points were used with the final vibrational matrices of dimension 1500. Rotational problem was solved using matrices of dimension $500(J+1-p)$, where $p$ is the rotationless parity. Atomic masses were used allowing approximately account for non-adiabatic effects. Calculations considered all transitions with $J \leq 60$. Lines with frequencies between $0 \mathrm{~cm}^{-1}$ and $4930 \mathrm{~cm}^{-1}$ are retained. The resulting line list consists of 78024 lines with intensities stronger than $10^{-24}$ in HITRAN units (cm/molecule) at 296 K. Partition function for ozone molecule was taken from work of Gamache et al. [30]. All ${ }^{16} \mathrm{O}_{3}$ intensities given in the present manuscript and supplementary materials are in natural abundance (scaled by 0.9929). Previously the calculations of the lines down to $10^{-32}$ intensities were retained and we saved these lines for wavenumbers below $2024 \mathrm{~cm}^{-1}$. This second line list will be used in the section 4 for taking into account resonances between levels affecting line intensities in the main line list.

Variational calculation results in the energy levels, line positions and line intensities. Every energy level is labelled by the rotational quantum number $J$, its parity $p$, and the number within each $(J, p)$ block. For spectroscopic 
and database purposes, however, labelling of the energy levels by asymmetric top rotational quantum numbers $J, K_{a}$ and $K_{c}$ as well as normal-mode vibrational quantum numbers $v_{1}, v_{2}$ and $v_{3}$ is important. Such labelling is performed in the following section.

\section{Matching of effective Hamiltonian and variational line lists}

Variational line lists, such as the ones described in the previous section, have been shown to give excellent predicted transition intensities, in some cases with the accuracy better than the experimental one [31,32,33]; however they have two major drawbacks. The accuracy of the line positions are at best an order of magnitude worse than the experimental value. Secondly there is no automatic line assignment with quantum numbers $K_{a}, K_{c}, v_{1}$, $v_{2}$ and $v_{3}$. EHs solve both these problems, but generally do not predict intensities with the required accuracy. A natural development is therefore lines lists synthesized using the two approaches, as has been done for $\mathrm{CO}_{2}$ $[34,35,36]$. However, while for $\mathrm{CO}_{2}$ combining the two approaches proved reasonably straightforward, for ${ }^{16} \mathrm{O}_{3}$ a procedure based only on direct line-byline matching is not sufficient. In the next two sections we develop a method for producing a synthesis of variational and EH line lists. This method results in a line list which combines the advantages and removes disadvantages of both its parents.

An effective Hamiltonian (EH) model is required to assign transitions from variational calculations but also to correct line intensities of transitions involving levels in resonance (see section 4). In the present work the HITRAN2016 database [12] has been used to assign all transitions from vari- 
ational line list except for 19 not found in HITRAN2016 but present in the JPL catalog [37].

Variational line list includes for each transition, upper and lower $J$ quantum numbers, line positions and intensities as well as energy of upper and lower states. In order to perform an accurate match with EH line list, several sets of criteria have been used. The primary criterion is based on identical upper and lower $J$ quantum numbers in both line lists. The second criterion is relative to lower energy states and line positions differences between transitions from the two line lists. Because of the presence of many nearby transitions, a third criterion based on intensities was included. For each transition from the variational line list, the corresponding transition is first searched in the EH line list using for second and third criterion low values for maximum differences on positions and lower energy state and close line intensities respectively. If not found, the line is searched again with less severe limit values for second or third criterion. By implementing eight different sets of various limits for second and third criteria, the corresponding transition is searched up to eight times in the EH line list. The main problem of such algorithm is that cases always exists where the selected sets of criteria will not lead to correct assignments due to resonance between levels involved in nearby transitions. As a consequences two different codes based on different sets of criteria were used, and assignments were compared and manually corrected in a few cases. Transitions can be particularly difficult to assign when they involve levels involved in a resonance. Indeed, in such cases variational calculation lead to erroneous intensities (see section 4) making assignments more difficult. For example, let us consider the two transitions (110-000; 
$\left.J, K_{a}, K_{c}=39238-40535\right)$ and (011-000, $\left.39534-40535\right)$ located in HITRAN [12] at 1681.8382 and $1682.0075 \mathrm{~cm}^{-1}$ with intensity equal to $7.750 \times 10^{-24}$ and $8.192 \times 10^{-24} \mathrm{~cm} /$ molecule respectively. Both transitions have same lower states and close upper states in resonance (with identical $J$ quantum numbers). In the variational line list the two corresponding transitions can be identified at 1682.0389 and $1682.2739 \mathrm{~cm}^{-1}$ with intensities equal to $1.996 \times 10^{-24}$ and $1.279 \times 10^{-23} \mathrm{~cm} /$ molecule, respectively. In such situations automatic assignments are hazardous and manual operations are required.

The main goal in this step is to assign most of the transitions from the variational line list. When transitions involve levels under resonance (as in example above), the variational calculations may lead to large intensity errors. This situation has been observed for the water [25] and carbon dioxide molecules [34] and arises because the variational-computed eigenfunctions are not precise even when the PES is refined using the observed line positions. Artificially strong transitions can be generated by stealing intensity from real transition. In general, it is only the distribution of intensity between transitions involving levels in resonance that is badly calculated, while the sum of intensities is not affected. This is the case for the example given above; the sum of intensities of the 2 lines in HITRAN is equal to $1.594 \times 10^{-23}$ $\mathrm{cm} /$ molecule, close to the sum of ab initio intensities equal to $1.479 \times 10^{-23}$ $\mathrm{cm} /$ molecule.

Two variational line lists, used in the present study, were calculated using PES from Polyansky et al. [20] and DMS from Tyuterev et al. [21]. First one from 0 to $4930 \mathrm{~cm}^{-1}$ with an intensity cutoff of $10^{-24} \mathrm{~cm} / \mathrm{molecule}$, and 
another from $0-2024 \mathrm{~cm}^{-1}$ with an intensity cutoff of $10^{-32} \mathrm{~cm} /$ molecule. The first one has been completely assigned whereas the second one has been assigned only partially to improve corrections (see section 4). Since a line list from 0 to $4930 \mathrm{~cm}^{-1}$, including $5 \mu \mathrm{m}$ and $3 \mu \mathrm{m}$ regions, is of more interest even with higher intensity cutoff, the focus of the present work is on calculations between 0 and $4930 \mathrm{~cm}^{-1}$. Assignments were found for the 78028 transitions from 0 to $4930 \mathrm{~cm}^{-1}$ with the exception of 171 lines with intensities ranging from $10^{-23}$ to $10^{-24} \mathrm{~cm} /$ molecule. 19 out of these 171 lines could be found in the JPL line list [37]. Note that variational line lists (with both intensity cutoff) are limited to $J$ values below 60 . Some transitions with $J>60$ and with intensities greater than $10^{-24} \mathrm{~cm} /$ molecule can be found in HITRAN [12]; these are not included in the present line list.

\section{Transitions involving levels in resonance}

Due to resonance between levels, outliers appear in the variational calculations caused by the wrong intensity distribution between transitions involving levels in resonance. The case of transitions with artificially high intensity stealing in variational calculation is an extreme case of erroneous intensity distribution from variational calculation. Since levels in resonance can be easily predicted, the intensity of variational calculation can be corrected using EH calculations tuned to better reproduce intensity perturbations for transitions involving levels in resonance. The first step of the corrections applied to variational calculation is to select transitions involving levels in resonance, meaning levels of same symmetry, same $J$ and close energy $\left(<3 \mathrm{~cm}^{-1}\right)$. In most cases, only 2 transitions (involving levels in resonance for upper or lower 
state) are stealing intensity from each other. These two transitions belongs to different bands as in example from section 3 with (110-000, 39238 $40535)$ and (011-000, $39534-40535)$ transitions located in HITRAN [12] at 1681.8382 and $1682.0075 \mathrm{~cm}^{-1}$ with intensity equal to $7.750 \times 10^{-24}$ and $8.192 \times 10^{-24} \mathrm{~cm} /$ molecule respectively. In this example, upper levels of these transitions are in resonance. Correction of intensities is based on using the EH intensity distribution to rebalance the intensities from the variational calculation. In the case of the two lines cited as example above, the HITRAN intensity of the (110-000, $39238-40535)$ and (011-000, 395 34 - 40535 ) transitions corresponds to $48.6 \%$ and $51.4 \%$ of the HITRAN summed intensities. By using the sum of intensities of corresponding transitions in variational line list $\left(1.479 \times 10^{-23} \mathrm{~cm} /\right.$ molecule $)$ and the distribution from HITRAN, the (110-000, 39238 - 40535$)$ and (011-000, 39534 $40535)$ transitions have corrected intensities equal to $7.188 \times 10^{-24}$ and $7.602 \times 10^{-24} \mathrm{~cm} /$ molecule, respectively. The originally calculated intensities were $1.996 \times 10^{-24}$ and $1.279 \times 10^{-23} \mathrm{~cm} /$ molecule, respectively, for these two transitions.

It may happen that four transitions are coupled together through levels in resonance in both upper and lower states. Two examples are given in Table 1 with four transitions involving levels in resonance both for upper and lower states. Such cases are rare (concerning less than 200 transitions) and have been partially corrected by hand as shown in Table 1 . The first example involves 4 transitions involving 2 different levels in the upper and lower states. The correction is based on the transfer of intensity distribution from EH calculation to variational calculation, using the sum of intensities 
from variational calculation. It is particularly difficult to perform corrections when the correspondence is not complete between EH and variational calculations. As shown in Table 1 for second set of "coupled" transitions, the 110-010 transition is missing in HITRAN2016. Moreover, the intensity from variational calculation for this transition is $3 \times 10^{-28} \mathrm{~cm} /$ molecule, compared to a few $10^{-24} \mathrm{~cm} /$ molecule for the other "connected" transitions. In such case, the distribution of intensities inside the three HITRAN transitions is applied to the three corresponding transitions using the sum of intensities of these transitions. The complete selection of transitions (involving levels in resonance) is not possible because the variational and $\mathrm{EH}$ line list are generated using an intensity cutoff. As a consequence, some weak transitions may be missing inside the resonance configuration. To illustrate this effect, we first performed corrections based on the variational line list with $10^{-24}$ $\mathrm{cm} /$ molecule intensity cutoff. These corrections, called "first-order corrections", allowed us to correct most of the intensities where the variational line list gave outliers. However, when among 2 coupled transitions involving levels in resonance, one transition is missing in the variational line list because the intensity cutoff, no correction can be performed. "Second-order corrections" are then performed using variational line list (from 0 to $2024 \mathrm{~cm}^{-1}$ ) with an intensity cutoff equal to $10^{-32} \mathrm{~cm} /$ molecule. As shown in Table 1 for the first set of "connected" transitions, (030-001, 19317 - 185 14), a transition calculated with an intensity of $2.55 \times 10^{-25}$ completed the scheme of 4 connected transitions and allows a more rigorous correction for transitions below $2024 \mathrm{~cm}^{-1}$. 
Table 1: Correction of intensity distribution within four transitions involving levels in resonance in both upper and lower state. Positions are given in $\mathrm{cm}^{-1}$, and intensities $(S)$ are for natural abundance (0.9929) in cm/molecule. Number 1 corresponds to HITRAN2016 parameters, 2 to the variational calculation, and 3 refers to the corrected intensity. .

\begin{tabular}{rrrrrrr}
\hline Band & $J^{\prime} K_{a}^{\prime} K_{c}^{\prime}-J^{\prime \prime} K_{a}^{\prime \prime} K_{c}^{\prime \prime}$ & $\omega_{1} / \mathrm{cm}^{-1}$ & $S_{1}$ & $\omega_{2} / \mathrm{cm}^{-1}$ & $S_{2}$ & $S_{3}$ \\
\hline $101-001$ & $19217-18514$ & 1021.5008 & $2.020(-23)$ & 1021.487041 & $2.001(-23)$ & $1.961(-23)$ \\
$101-100$ & $19217-18216$ & 1021.5782 & $1.370(-22)$ & 1021.563043 & $1.392(-22)$ & $1.330(-22)$ \\
$030-001$ & $19317-18514$ & 1021.5989 & $1.123(-24)$ & 1021.877708 & $2.551(-25)$ & $1.090(-24)$ \\
$030-100$ & $19317-18216$ & 1021.6763 & $7.523(-24)$ & 1021.953711 & $1.554(-24)$ & $7.304(-24)$ \\
& & & & & & \\
$011-010$ & $491138-501139$ & 964.3651 & $5.221(-24)$ & 964.277996 & $5.314(-24)$ & $5.401(-24)$ \\
$101-001$ & $49148-50447$ & 961.6807 & $3.275(-24)$ & 961.530441 & $2.648(-24)$ & $3.388(-24)$ \\
$002-001$ & $49446-50447$ & 963.2390 & $7.839(-24)$ & 963.153542 & $8.937(-24)$ & $8.110(-24)$ \\
$110-010$ & $49 ? ?-501139$ & N.A & N.A & 962.213352 & $3.140(-28)$ & N.A \\
\hline \hline
\end{tabular}

In order to observe the effects of the corrections on the line intensities, ratios of line intensities (HITRAN2016 divided by variational calculation) before and after correction can be plotted. Figures 1 and 2 correspond to comparisons with HITRAN2016 respectively for 110-000 and 101-000 bands using various symbols according to cases: black symbols are used when no correc- 


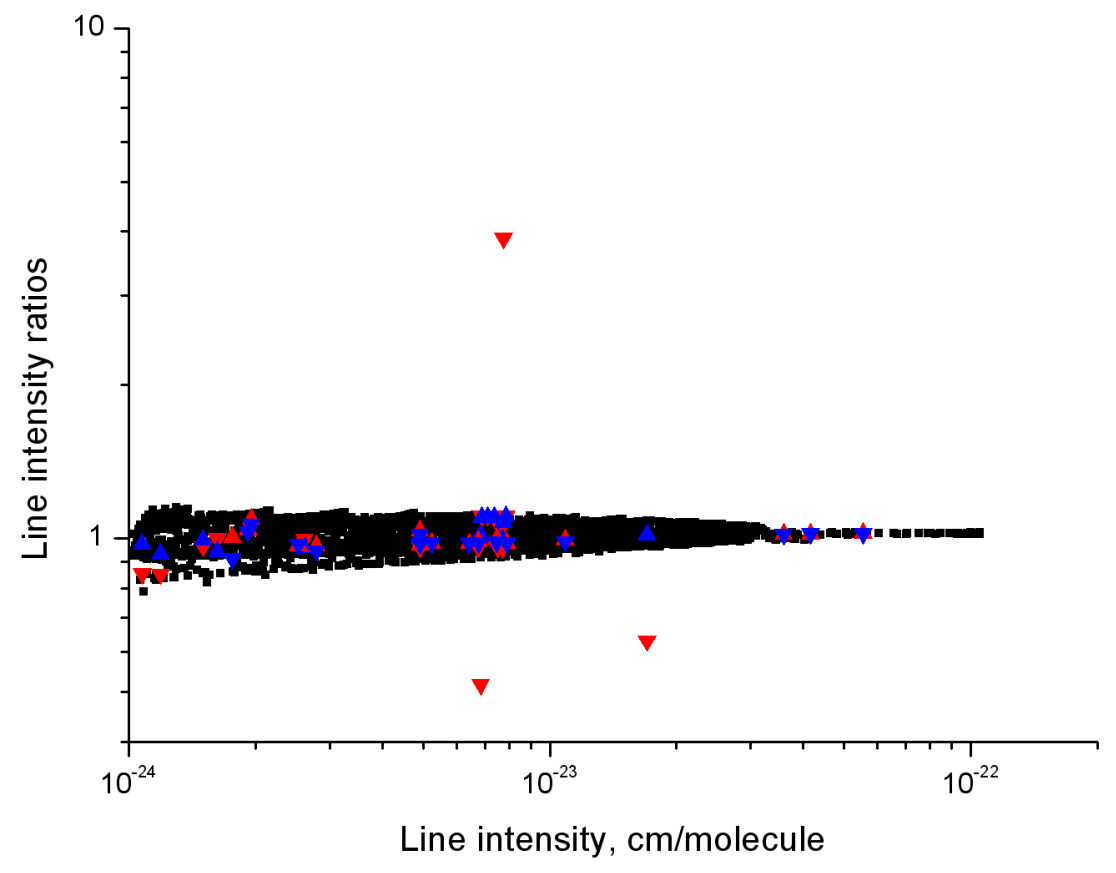

Figure 1: Ratios of line intensities (HITRAN2016/ab-initio) for 110-000 band obtained with and without corrections of line intensities: $\mathbf{\square}$ uncorrected; $\boldsymbol{\Delta}$ first-order correction; $\boldsymbol{\Delta}$ second-order correction; $\boldsymbol{\nabla}$ uncorrected transition in resonance. 


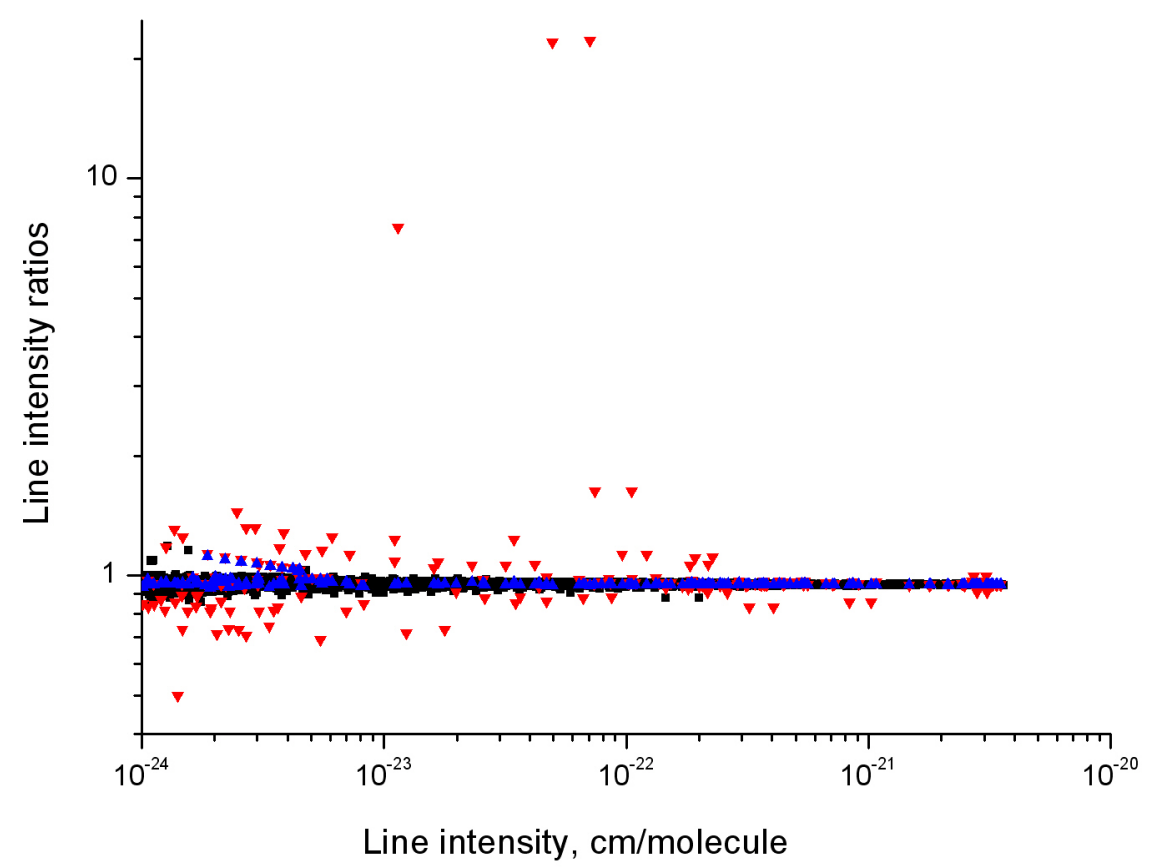

Figure 2: Ratios of line intensities (HITRAN2016/ab-initio) for 101-000 band obtained with and without corrections of line intensities: $\mathbf{\square}$ uncorrected; $\boldsymbol{\Delta}$ first-order correction; $\boldsymbol{\Delta}$ second-order correction; $\boldsymbol{\nabla}$ uncorrected transition in resonance. 
tion is performed (because transition involves no level in resonance), blue up triangles show when "first-order corrections" are applied (red down triangles are corresponding uncorrected variational intensities), and blue down triangles when "second-order corrections" are applied (in red up triangles when variational intensities are not corrected). As can be observed in Fig. 1 for 110-000 band, the red symbol corresponding to ratio of about 3.9 is clearly an outlier. It corresponds to the (110-000, 39238 - 405 35) transition for which upper level is in resonance with upper level of (011-001, 395 34 - 40535$)$ transition. After correction based on the transfer of intensity distribution among these two transitions from EH to variational calculation, the corresponding blue up triangle symbol for (110-000, 39238 - 40535$)$ transition corresponds to a ratio equal to 1.08 instead of 3.6. In the same way the correction for 011-001, $39534-40535$ ) transition lead to a ratio of 1.08 instead of 0.64 (when using ab initio intensity without correction). As can be seen in Figs. 1 and 2, the several red symbols that looked like outliers correspond to blue symbols closer to average ratio. For bands located above $2024 \mathrm{~cm}^{-1}$ no "second order correction" was performed since no variational calculation were available with intensity cutoff lower than $10^{-24}$ $\mathrm{cm} /$ molecule. An example for the 111-010 band is given in Fig. 3 where several black symbols still appear to be outliers. Only a few lines have been corrected using "second-order corrections", and all the black symbols looking as outliers correspond to transition with wavenumbers higher than 2024 $\mathrm{cm}^{-1}$. By using variational calculation with low intensity cutoff on the whole 0-5000 $\mathrm{cm}^{-1}$ spectral region, we believe new resonances between levels will be identified and will allow improved intensities for weak transitions $\left(<10^{-22}\right)$ 


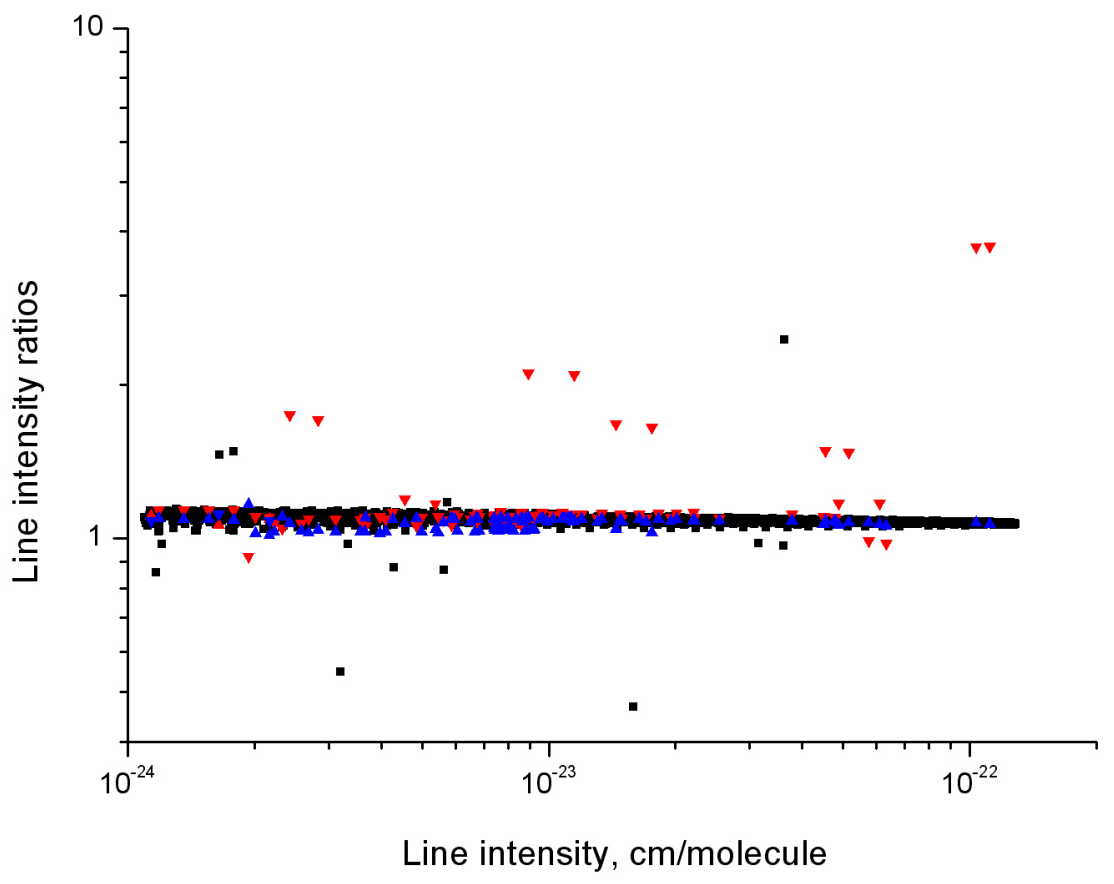

Figure 3: Ratios of line intensities (HITRAN2016/ab-initio) for 111-010 band obtained

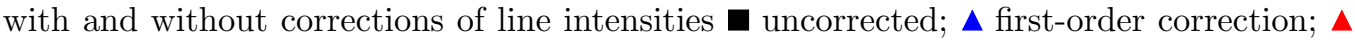
second-order correction; $\boldsymbol{\nabla}$ uncorrected transition in resonance.

above $2024 \mathrm{~cm}^{-1}$.

The whole variational line list both without correction and with "firstand second-order corrections" is given as supplementary material. This file also contains full vibrational and rotational assignments. 


\section{Comparison of the resulting line list with experimental obser- vations and effective Hamiltonian calculations}

Systematic comparisons of variational line intensities (with the corrections of section 4) with recent calculated and measured line intensities have been performed in order to better understand issues concerning ozone line intensities. Ratios of variational line intensities to intensities from the literature have been plotted band by band and are available in supplementary material. For each band, ratios using measurements or calculations from literature have been distinguished. The comparisons for 001-band are presented in Figs. 4 and 5. For calculations from literature, following data have been used: EH calculations present in HITRAN2016 [12], in JPL catalog [37], and performed recently by Flaud [38] (denoted JMF hereafter) and by Barbe and Tyuterev (denoted URCA-ESA) [10], as well as ab initio calculations from Tyuterev et al. [21]. As for experimental measurements, recent studies from LERMA [22, 23], DLR [10], and Barbe and De Backer [39] (noted GSMA hereafter) have been selected to be compared with present variational calculations and synthetic line list. A sample of average ratios and associated SDs are presented in Table 2 for various bands in the microwave, $14 \mu \mathrm{m}, 10 \mu \mathrm{m}$ and $5 \mu \mathrm{m}$ spectral regions. The whole set of average ratios for around 100 bands between 0 and $5000 \mathrm{~cm}^{-1}$ is given in supplementary material.

For rotational bands, comparisons between our variational (synthetic) line list and HITRAN2016 [12] from one side and JPL [37, 18] from other side lead to a general trend (see Table 2) where variational intensities are closer to JPL than to HITRAN2016. Agreement between variational line list and JPL catalog is excellent for the 2 most intense $000-000$ and 010- 
010 bands. The average discrepancy is sub-percent with a SD of around 0.5\% (see Table 2). For these two bands, HITRAN2016 line intensities are systematically lower by $4 \%$ as compared to variational and JPL line lists. For other bands, when HITRAN 2016 line intensities are systematically lower by around $5 \%$ as compared to variational line list whereas JPL line intensities are systematically higher than variational intensities by 1-5\% depending on the bands. For those bands variational intensities are between HITRAN2016 and JPL intensities.

In the $14 \mu \mathrm{m}$ spectral region, revisited intensities from Birk et al.[40] led to systematic scaling intensities in this region by $1.4 \%$ as compared to HITRAN [12]. This correction lead to closer intensities but are still lower than variational intensities by around 4-5\% for the two strongest bands presented in Table 2. 


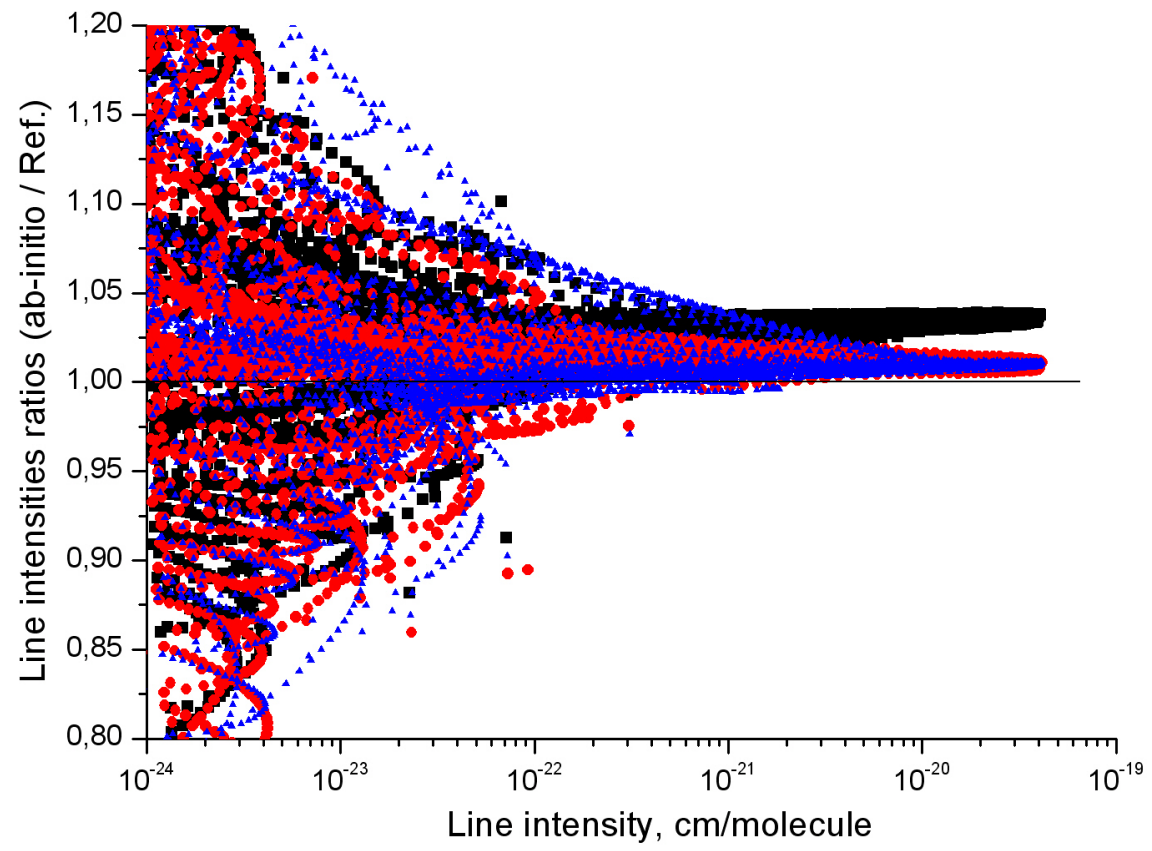

Figure 4: Ratios of variational intensities to effective Hamiltonian intensities from HI-

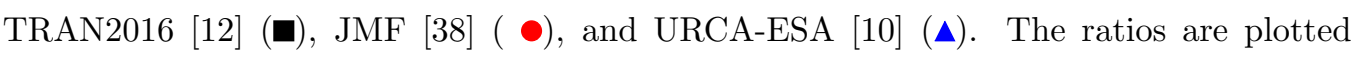
versus line intensity in a log scale. 


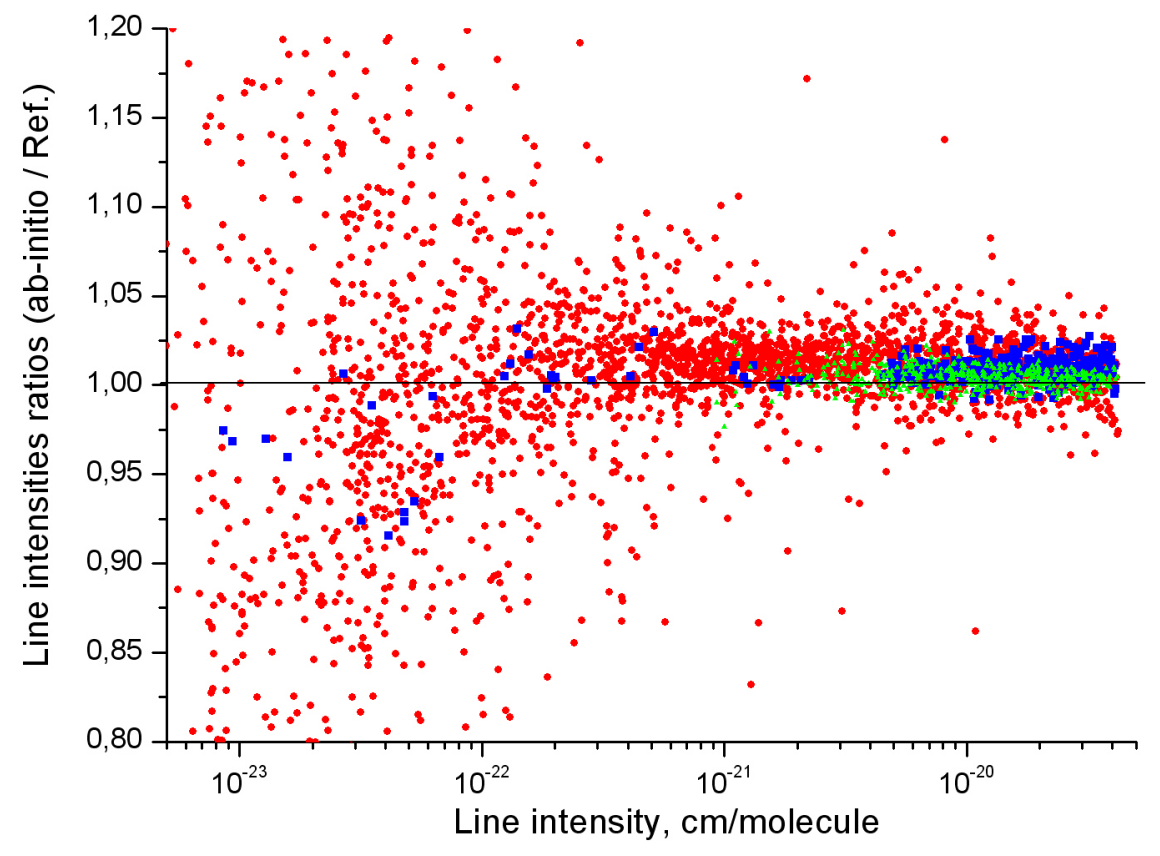

Figure 5: Ratios of variational intensities to measured intensities from DLR [10] (•), GSMA [39] (ロ), and LERMA [22, 23] ( $\mathbf{\Delta})$. The ratios are plotted versus line intensity in a log scale. 
Table 2: Extract of average comparisons between intensities from variational calculation and intensities from literature for various bands in the microwave, $14 \mu \mathrm{m}, 10$ $\mu$ mand $5 \mu \mathrm{m}$ regions. $R_{1}$ (ab-initio/HITRAN) and $R_{2}$ (ab-initio/Ref) are average ratios of line intensities. Digits between parentheses correspond to the standard deviation of the average in units of the last digit. $\sigma_{\min }$ and $\sigma_{\max }$ correspond the range of positions in $\mathrm{cm}^{-1}, S_{\max }$ is the maximum line intensity (given with powers of 10 in parenthesis) in the band ( $\mathrm{cm} /$ molecule). Common transitions between variational calculation and HITRAN $\left(N_{1}\right)$ and between variational calculation and reference $\left(N_{2}\right)$ are given.

\begin{tabular}{rrrrrrrrr}
\hline$\sigma_{\min }$ & $\sigma_{\max }$ & $S_{\max }$ & Band & $N_{1}$ & $R_{1}$ & $N_{2}$ & $R_{2}$ & Ref \\
\hline 2.5 & 176.4 & $1.43(-21)$ & $000-000$ & 3088 & $1.0399(54)$ & 3075 & $1.0013(57)$ & $\mathrm{JPL}$ \\
5.6 & 146.2 & $4.65(-23)$ & $010-010$ & 1460 & $1.0404(41)$ & 1460 & $1.0051(29)$ & $\mathrm{JPL}$ \\
14.8 & 121.5 & $6.44(-24)$ & $100-100$ & 601 & $1.0482(158)$ & 598 & $0.9724(43)$ & $\mathrm{JPL}$ \\
12.5 & 125.1 & $8.78(-24)$ & $001-001$ & 723 & $1.0471(172)$ & 722 & $0.9892(341)$ & $\mathrm{JPL}$ \\
43.9 & 94.5 & $1.52(-24)$ & $020-020$ & 125 & $1.0558(100)$ & 105 & $0.9531(9)$ & $\mathrm{JPL}$ \\
24.2 & 103.3 & $3.80(-24)$ & $100-001$ & 66 & $1.0444(224)$ & 40 & $0.9508(1208)$ & $\mathrm{JPL}$ \\
22.6 & 103.9 & $4.10(-24)$ & $001-100$ & 69 & $1.0462(166)$ & 38 & $0.9858(467)$ & $\mathrm{JPL}$
\end{tabular}

Continued on next page 


\begin{tabular}{rrrrrrrrr}
\hline$\sigma_{\min }$ & $\sigma_{\max }$ & $S_{\max }$ & Band & $N_{1}$ & $R_{1}$ & $N_{2}$ & $R_{2}$ & Ref \\
\hline 574.2 & 873.6 & $1.66(-21)$ & $010-000$ & 4840 & $1.0687(225)$ & 4592 & $1.0545(247)$ & BirkPC \\
592.8 & 832.0 & $1.10(-22)$ & $020-010$ & 2794 & $1.0525(139)$ & 2770 & $1.0385(148)$ & BirkPC \\
& & & & & & & & \\
961.7 & 1226.0 & $4.06(-20)$ & $001-000$ & 4877 & $1.0244(613)$ & 476 & $1.0041(63)$ & LERMA \\
975.5 & 1213.8 & $2.13(-23)$ & $200-001$ & 1082 & $1.2817(346)$ & 1068 & $1.0335(213)$ & JMF \\
& & & & & & & & \\
1960.7 & 2244.0 & $3.60(-21)$ & $101-000$ & 3180 & $1.0510(166)$ & 316 & $1.0059(42)$ & LERMA \\
1983.5 & 2066.3 & $4.76(-23)$ & $102-001$ & 1286 & $0.8898(498)$ & & & \\
2011.0 & 2108.2 & $1.28(-22)$ & $111-010$ & 1634 & $0.9186(491)$ & & & \\
1915.2 & 2104.1 & $4.86(-23)$ & $012-010$ & 682 & $0.8445(748)$ & & & \\
2052.3 & 2133.3 & $1.70(-22)$ & $030-000$ & 25 & $1.0919(1610)$ & & & \\
\hline \hline
\end{tabular}

In the $10 \mu \mathrm{m}$ spectral region, two bands have been given as example in Table 2, the strong 001-000 band and a weaker band, namely the 200-001 band, for which a large dispersion is observed when comparing variational and HITRAN2016 intensities. For the 001-000 band a deviation of around $2.5 \%$ is observed between variational and HITRAN intensities, whereas an average sub-percent agreement is observed between LERMA measurements (476 strong transitions) and variational calculation with a SD of $0.6 \%$. For the weak 200-001 band plotted in Fig. 6, the average deviation between variational and HITRAN intensities reaches $28 \%$. Let us recall that in this region HITRAN2016 is based on EH from Ref. [41] where no measurements were available for this band. With recent measurements from Birk et al.[10], Flaud's calculation [38] leads to intensities in better agreement with varia- 


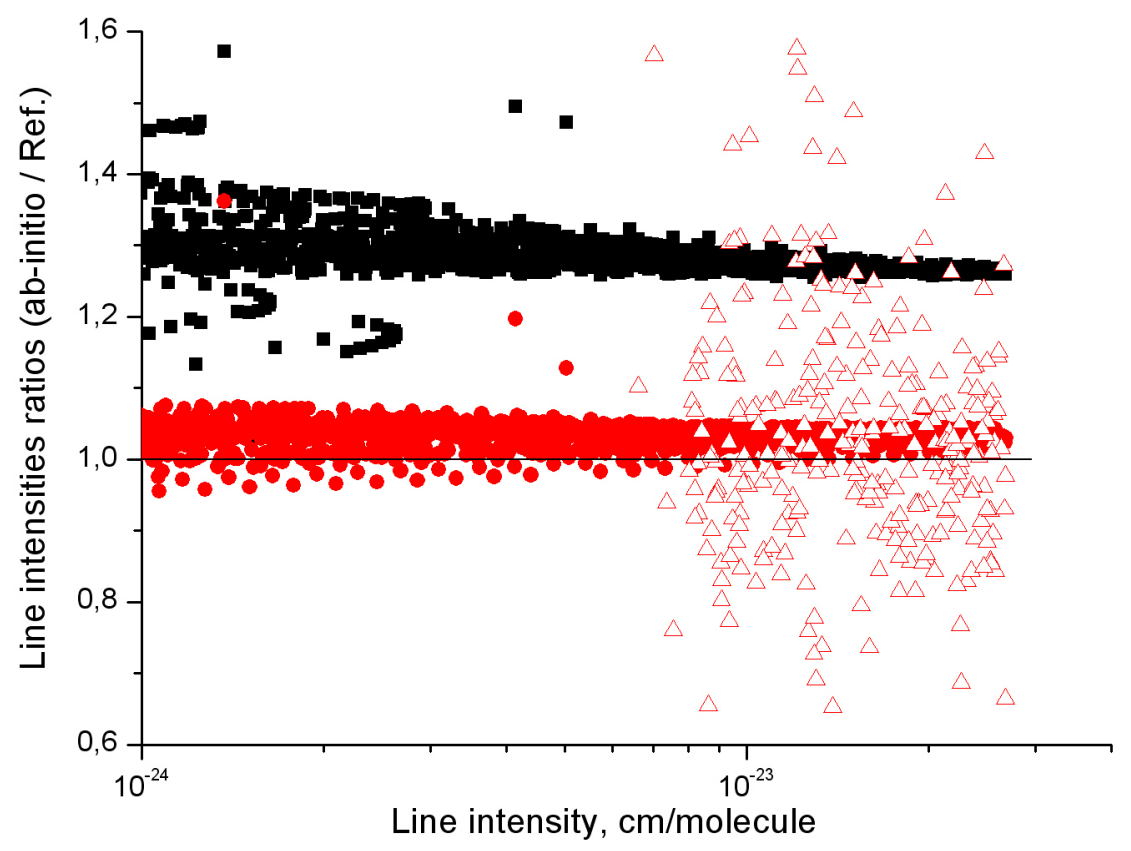

Figure 6: Ratios of variational intensities to effective Hamiltonian calculated intensities from HITRAN2016 (black squares) and from Flaud [38] (solid red circles) as well as to measured intensities from DLR [10] (open red triangles) for the 200-001 band.

tional calculation (deviation of 3.3\%). Without experimental measurements Hamiltonian calculated intensities can deviate by up to $30 \%$ whereas variational calculation (that does not require measurements) lead to intensities quite close $(3.3 \%)$ to those obtained from $\mathrm{EH}$ using new measurements for this band.

In the $5 \mu \mathrm{m}$ spectral region, for the strongest 101-000 band, the average agreement between LERMA measurements and variational line list is equal to $0.6 \%$ for 316 intense common transitions with a SD of $0.4 \%$. However, 
a systematic deviation between HITRAN2016 and variational intensities of around $5 \%$ is observed with a SD of $1.7 \%$ for 3180 common transitions. The other four bands at $5 \mu \mathrm{m}$ presented in Table 2 are bands which present average deviation up to $15 \%$ when comparing HITRAN2016 and variational intensities. Those bands were calculated from Hamiltonian calculation without fitting any measurements for these bands from literature. It would be very interesting to measure (even with accuracy of $2-3 \%$ which is more realistic accuracy for the weak bands, than the sub-percent accuracy) transitions of these bands in order to check the variational calculation. The case of 200001 band, where the disagreement between HITRAN2016 and variational linelist is about $28 \%$ and the agreement between variational calculations and $\mathrm{EH}$ calculations based on the fitting to the experimental data is only 3 $\%$ is very promising in this respect. This case indicates, that the reason of very serious disagreement between HITRAN16 and variational calculations for certain bands might point to the problems with the present HITRAN2016 version.

\section{Conclusions}

Very accurate intensity calculations from the first principles and determination of the line positions with the accuracy of experiment by effective Hamiltonian calculations do not provide necessary final result for the spectroscopic databases in their separate essences. The variational calculations provide the line centers with the accuracy far from the experimental values. The intensities computed using an effective Hamiltonian (EH) do not provide

accurate values beyond the ones included in the empirical determination of 
the effective dipole constants.

A method for constructing a synthetic line list is developed. Matching lines between the variationally-calculated and EH line lists is combined with the removal of outliers of such a line list due to the distorting effects of resonances in the variational calculations which can be associated with the imperfect accuracy of its line positions and its underlying potential energy surface. This method could be useful in cases when the synthesis of variational and EH linelists is required due to complimentary advantages and disadvantages of both of them. We are now considering other molecules where this approach may be useful.

Our newly constructed synthetic line list gives line positions with the accuracy of experiment. Comparison of the ab initio calculated intensities with the experimentally available data also demonstrates the accuracy of the line intensities which are predicted within the experimental error for most of the bands. The most stunning difference in accuracy of the calculated intensity arises for the 200-001 band. The line intensities of this band was calculated using both EHs and variational calculations before the experimentally adjusted $\mathrm{EH}$ become available. The EH predictions deviated from EH calculations adjusted to the measurements by $28 \%$ while the predictions of the variational calculations deviated by only $3 \%$, which seems a realistic estimation of the measurement uncertainty. The synthesis of the $\mathrm{EH}$ and variational calculations presented here provides the information required to represent the ${ }^{16} \mathrm{O}_{3}$ spectrum in atmospheric databases such as HITRAN and GEISA.

Further work is required to calculate the lines with higher rotational quan- 
tum numbers $J$. However, before this further improvement in the accuracy of the fitted semi-empirical PES should result in better line intensities and fewer outliers. Isotopologues of ozone also require the similar type calculations for the completeness of the natural abundance ozone linelist. All these improvements are under way.

\section{Acknowledgment}

We thanks Geoff Toon (JPL) for sharing his assessment of the HITRAN 2016 ozone line list with us and for helpful discussions. The project started with LERMA measurements performed by Christof Janssen and co-workers, we thank them for sharing their results prior to publication. This work was supported by the UK Natural Environment Research Council under grants NE/N001508/1 and the European Research Council under ERC Advanced Investigator grant 8838302 . VYuM, NFZ and OLP acknowledge support by State Project IAP RAS No.0035-2019-0016.

\section{References}

[1] S. Solomon, Stratospheric ozone depletion: A review of concepts and history, Rev. Geophys. 37 (1999) 275-316. doi:10.1029/1999RG900008.

[2] C. Viatte, M. Schneider, A. Redondas, F. Hase, M. Eremenko, P. Chelin, J. M. Flaud, T. Blumenstock, J. Orphal, Comparison of ground-based FTIR and Brewer O-3 total column with data from two different IASI algorithms and from OMI and GOME-2 satellite instruments, Atmos. Meas. Tech. 4 (3) (2011) 535-546. doi:10.5194/amt-4-535-2011. 
[3] A. Boynard, D. Hurtmans, M. E. Koukouli, F. Goutail, J. Bureau, S. Safieddine, C. Lerot, J. Hadji-Lazaro, C. Wespes, J.-P. Pommereau, A. Pazmino, I. Zyrichidou, D. Balis, A. Barbe, S. N. Mikhailenko, D. Loyola, P. Valks, M. Van Roozendael, P.-F. Coheur, C. Clerbaux, Seven years of IASI ozone retrievals from FORLI: validation with independent total column and vertical profile measurements, Atmos. Meas. Tech. 9 (9) (2016) 4327-4353. doi:10.5194/amt-9-4327-2016.

[4] J.-M. Flaud, C. Camy-Peyret, C. P. Rinsland, M. A. H. Smith, M. V. Devi, Atlas of ozone spectral parameters from microwave to medium infrared, Academic, San Diego, CA, 1990.

[5] G. Wagner, M. Birk, F. Schreier, J. M. Flaud, Spectroscopic database for ozone in the fundamental spectral regions, J. Geophys. Res. Atmos. 107 (D22) (2002). doi:10.1029/2001JD000818.

[6] C. P. Rinsland, J. M. Flaud, A. Perrin, A. Birk, G. Wagner, A. Goldman, A. Barbe, M. R. De Backer-Barilly, S. N. Mikhailenko, V. G. Tyuterev, M. A. H. Smith, V. M. Devi, D. C. Benner, F. Schreier, K. V. Chance, J. Orphal, T. M. Stephen, Spectroscopic parameters for ozone and its isotopes: recent measurements, outstanding issues, and prospects for improvements to HITRAN, J. Quant. Spectrosc. Radiat. Transf. 82 (14) (2003) 207-218. doi:10.1016/S0022-4073(03)00154-7.

[7] J.-M. Flaud, C. Piccolo, B. Carli, A. Perrin, L. H. Coudert, J.-L. Teffo, L. R. Brown, Molecular line parameters for the MIPAS (Michelson Interferometer for Passive Atmospheric Sounding) experiment, Atmos. Oceanic Opt. 16 (2003) 172-182. 
[8] Y. Babikov, S. N. Mikhailenko, A. Barbe, V. G. Tyuterev, S\&MPO - An information system for ozone spectroscopy on the WEB, J. Quant. Spectrosc. Radiat. Transf. 145 (2014) 169-196. doi:10.1016/j.jqsrt.2014.04.024.

[9] C. Janssen, C. Boursier, P. Jeseck, Y. Te, Line parameter study of ozone at 5 and $10 \mu \mathrm{m}$ using atmospheric FTIR spectra from the ground: A spectroscopic database and wavelength region comparison, J. Mol. Spectrosc. 326 (2016) 48-59. doi:10.1016/j.jms.2016.04.003.

[10] M. Birk, G. Wagner, A. Barbe, M.-R. De Backer, M. Rotger, ESA SEOM-IAS - Measurement and line parameter database O3 MIR region (Version 1) [Data set], Zenodo (2018). doi:10.5281/zenodo.1492543.

[11] A. Noelle, G. K. Hartmann, A. Fahr, D. Lary, Y.-P. Lee, P. LimaoVieira, R. Locht, F. J. Martin-Torres, K. McNeill, J. J. Orlando, A.C. V. F. Salama, R. P. Wayne, M. Brunger, UV/Vis+ Spectra Data Base, 11th Edition (2017).

[12] I. E. Gordon, L. S. Rothman, C. Hill, R. V. Kochanov, Y. Tan, P. F. Bernath, M. Birk, V. Boudon, A. Campargue, K. V. Chance, B. J. Drouin, J.-M. Flaud, R. R. Gamache, J. T. Hodges, D. Jacquemart, V. I. Perevalov, A. Perrin, K. P. Shine, M.-A. H. Smith, J. Tennyson, G. C. Toon, H. Tran, V. G. Tyuterev, A. Barbe, A. G. Császár, V. M. Devi, T. Furtenbacher, J. J. Harrison, J.-M. Hartmann, A. Jolly, T. J. Johnson, T. Karman, I. Kleiner, A. A. Kyuberis, J. Loos, O. M. Lyulin, S. T. Massie, S. N. Mikhailenko, N. Moazzen-Ahmadi, H. S. P. Müller, 
O. V. Naumenko, A. V. Nikitin, O. L. Polyansky, M. Rey, M. Rotger, S. W. Sharpe, K. Sung, E. Starikova, S. A. Tashkun, J. Vander Auwera, G. Wagner, J. Wilzewski, P. Wcisło, S. Yu, E. J. Zak, The HITRAN 2016 molecular spectroscopic database, J. Quant. Spectrosc. Radiat. Transf. 203 (2017) 3-69. doi:10.1016/j.jqsrt.2017.06.038.

[13] N. Jacquinet-Husson, R. Armante, N. A. Scott, A. Chédin, L. Crépeau, C. Boutammine, A. Bouhdaoui, C. Crevoisier, V. Capelle, C. Boonne, N. Poulet-Crovisier, A. Barbe, D. C. Benner, V. Boudon, L. R. Brown, J. Buldyreva, A. Campargue, L. H. Coudert, V. M. Devi, M. J. Down, B. J. Drouin, A. Fayt, C. Fittschen, J.-M. Flaud, R. R. Gamache, J. J. Harrison, C. Hill, Ø. Hodnebrog, S. M. Hu, D. Jacquemart, A. Jolly, E. Jiménez, N. N. Lavrentieva, A. W. Liu, L. Lodi, O. M. Lyulin, S. T. Massie, S. Mikhailenko, H. S. P. Müller, O. V. Naumenko, A. Nikitin, C. J. Nielsen, J. Orphal, V. I. Perevalov, A. Perrin, E. Polovtseva, A. Predoi-Cross, M. Rotger, A. A. Ruth, S. S. Yu, K. Sung, S. A. Tashkun, J. Tennyson, V. G. Tyuterev, J. Vander Auwera, B. A. Voronin, A. Makie, The 2015 edition of the GEISA spectroscopic database, J. Mol. Spectrosc. 327 (2016) 31-72. doi:10.1016/j.jms.2016.06.007.

[14] M. A. H. Smith, A. M. Devi, D. C. Benner, The quest for ozone intensities in the 9-11 $\mu$ m region: A retrospective, J. Quant. Spectrosc. Radiat. Transf. 113 (2012) 825-828. doi:10.1016/j.jqsrt.2012.02.027.

[15] N. Glatthor, T. von Clarmann, G. P. Stiller, M. Kiefer, A. Laeng, B. M. Dinelli, G. Wetzel, J. Orphal, Differences in ozone retrieval in MIPAS 
channels A and AB: a spectroscopic issue, Atmos. Meas. Tech. 11 (8) (2018) 4707-4723. doi:10.5194/amt-11-4707-2018.

[16] A. Campargue, S. Kassi, D. Mondelain, A. Barbe, E. Starikova, M. R. De Backer, V. G. Tyuterev, Detection and analysis of three highly excited vibrational bands of O-16(3) by CW-CRDS near the dissociation threshold, J. Quant. Spectrosc. Radiat. Transf. 152 (2015) 84-93. doi:10.1016/j.jqsrt.2014.10.019.

[17] B. J. Drouin, T. J. Crowford, S. Yu, Validation of ozone intensities at $10 \mu \mathrm{m}$ with THz spectrometry, J. Quant. Spectrosc. Radiat. Transf. 203 (2017) 282-292. doi:10.1016/j.jqsrt.2017.06.035.

[18] M. Birk, G. Wagner, I. E. Gordon, B. J. Drouin, Ozone intensities in the rotational bands, J. Quant. Spectrosc. Radiat. Transf. 226 (2019) 60-65. doi:10.1016/j.jqsrt.2019.01.004.

[19] V. G. Tyuterev, R. V. Kochanov, S. A. Tashkun, Accurate ab initio dipole moment surfaces of ozone: First principle intensity predictions for rotationally resolved spectra in a large range of overtone and combination bands, J. Chem. Phys. 146 (2017) 064304. doi:10.1063/1.4973977.

[20] O. L. Polyansky, N. F. Zobov, I. I. Mizus, A. A. Kyuberis, L. Lodi, J. Tennyson, Potential energy surface, dipole moment surface and the intensity calculations for the $10 \mu \mathrm{m}, 5 \mu \mathrm{m}$ and $3 \mu \mathrm{m}$ bands of ozone, J. Quant. Spectrosc. Radiat. Transf. 210 (2018) 127-135. doi:10.1016/j.jqsrt.2018.02.018. 
[21] V. G. Tyuterev, A. Barbe, D. Jacquemart, C. Janssen, S. N. Mikhailenko, E. N. Starikova, Ab initio predictions and laboratory validation for consistent ozone intensities in the MW, 10 and $5 \mathrm{~m}$ ranges, J. Chem. Phys. 150 (2019) 184303. doi:10.1063/1.5089134.

[22] C. Janssen, C. Boursier, H. Elandalouss, P. Jeseck, P. Marie-Jeanne, Y. Té, D. Jacquemart, F. Thibout, M. Vaudescal-Escudier, Multispectral investigation of ozone : Part I. Setup and uncertainty budget, JQSRT This issue.

[23] D. Jacquemart, C. Boursier, H. Elandaloussi, M. Vaudescal-Escudier, P. Jeseck, Y. Té, F. Thibout, C. Janssen, Multi-spectral investigation of ozone: Part II. Line intensities at $5 \mu \mathrm{m}$ and $10 \mu \mathrm{m}$ at one percent accuracy, JQSRT This issue.

[24] J. Tennyson, M. A. Kostin, P. Barletta, G. J. Harris, O. L. Polyansky, J. Ramanlal, N. F. Zobov, DVR3D: a program suite for the calculation of rotation-vibration spectra of triatomic molecules, Comput. Phys. Commun. 163 (2004) 85-116.

[25] L. Lodi, J. Tennyson, Line lists for $\mathrm{H}_{2}{ }^{18} \mathrm{O}$ and $\mathrm{H}_{2}{ }^{17} \mathrm{O}$ based on empirically-adjusted line positions and ab initio intensities, J. Quant. Spectrosc. Radiat. Transf. 113 (2012) 850-858. doi:10.1016/j.jqsrt.2012.02.023.

[26] L. Lodi, J. Tennyson, Theoretical methods for small-molecule rovibrational spectroscopy, J. Phys. B: At. Mol. Opt. Phys. 43 (2010) 133001. 
[27] J. Tennyson, Accurate variational calculations for line lists to model the vibration rotation spectra of hot astrophysical atmospheres, WIREs Comput. Mol. Sci. 2 (2012) 698-715. doi:10.1002/wcms.94.

[28] H.-J. Werner, P. J. Knowles, G. Knizia, F. R. Manby, M. Schütz, Molpro: a general-purpose quantum chemistry program package, WIREs Comput. Mol. Sci. 2 (2012) 242-253. doi:10.1002/wcms.82.

[29] I. I. Mizus, A. A. Kyuberis, N. F. Zobov, V. Y. Makhnev, O. L. Polyansky, J. Tennyson, High accuracy water potential energy surface for the calculation of infrared spectra, Phil. Trans. Royal Soc. London A 376 (2018) 20170149. doi:10.1098/rsta.2017.0149.

[30] R. R. Gamache et al., Total Internal Partition Sums for 167 isotopologues of 53 molecules important in planetary atmospheres: application to HITRAN2016 and beyond, J. Quant. Spectrosc. Radiat. Transf. 203 (2017) 70-87. doi:10.1016/j.jqsrt.2017.03.045.

[31] O. L. Polyansky, K. Bielska, M. Ghysels, L. Lodi, N. F. Zobov, J. T. Hodges, J. Tennyson, High accuracy $\mathrm{CO}_{2}$ line intensities determined from theory and experiment, Phys. Rev. Lett. 114 (2015) 243001. doi:10.1103/PhysRevLett.114.243001.

[32] T. Odintsova, E. Fasci, L. Moretti, E. J. Zak, O. L. Polyansky, J. Tennyson, L. Gianfrani, A. Castrillo, Highly-accurate intensity factors of pure $\mathrm{Co}_{2}$ lines near $2 \mu \mathrm{m}$, J. Chem. Phys. 146 (2017) 244309. doi:10.1063/1.4989925. 
[33] D. Long, Z. Reed, A. Fleisher, J. Mendonca, S. Roche, J. Hodges, Highaccuracy near-infrared carbon dioxide intensity measurements to support remote sensing, Geophys. Res. Lett. 47 (2020) e2019GL086344. doi:10.1029/2019GL086344.

[34] E. J. Zak, J. Tennyson, O. L. Polyansky, L. Lodi, S. A. Tashkun, V. I. Perevalov, A room temperature $\mathrm{CO}_{2}$ line list with ab initio computed intensities, J. Quant. Spectrosc. Radiat. Transf. 177 (2016) 31-42. doi:10.1016/j.jqsrt.2015.12.022.

[35] E. J. Zak, J. Tennyson, O. L. Polyansky, L. Lodi, N. F. Zobov, S. A. Tashkun, V. I. Perevalov, Room temperature line lists for $\mathrm{CO}_{2}$ symmetric isotopologues with ab initio computed intensities, J. Quant. Spectrosc. Radiat. Transf. 189 (2017) 267 - 280. doi:10.1016/j.jqsrt.2016.11.022.

[36] E. J. Zak, J. Tennyson, O. L. Polyansky, L. Lodi, N. F. Zobov, S. A. Tashkun, V. I. Perevalov, Room temperature line lists for $\mathrm{CO}_{2}$ asymmetric isotopologues with $a b$ initio computed intensities, J. Quant. Spectrosc. Radiat. Transf. 203 (2017) 265-281. doi:10.1016/j.jqsrt.2017.01.037.

[37] H. M. Pickett, R. L. Poynter, E. A. Cohen, M. L. Delitsky, J. C. Pearson, H. S. P. Müller, Submillimeter, millimeter, and microwave spectral line catalog, J. Quant. Spectrosc. Radiat. Transf. 60 (1998) 883-890.

[38] J. M. Flaud, Effective Hamiltonian calculation at $10 \mu \mathrm{m}$, private communication (2019). 
[39] A. Barbe, M.-R. De Backer, Measurements of line positions and intensities of $\nu_{1}$ and $\nu_{3}$ transitions, private communication (2019).

[40] M. Birk, G. Wagner, Rescaled intensities at $14 \mu \mathrm{m}$ region, private communication (2019).

[41] J.-M. Flaud, G. Wagner, M. Birk, C. Camy-Peyret, M. R. DebackerBarilly, A. Barbe, C. Piccollo, Ozone absorption around $10 \mu \mathrm{m}$, J. Geophys. Res. 108 (2003). doi:10.1029/2002JD002755. 